\title{
Pan-Facial Fractures: A Retrospective Study and Review of Literature
}

\author{
Abdeljalil Abouchadi, Hind Taoufik, Oussama Nacir, Adil Arrob \\ Department of Maxillo Facial, Stomatology and Plastic Surgery, Avicenne Military Teaching Hospital, Marrakech, Morocco \\ Email:pr_abouchadi@outlook.com
}

How to cite this paper: Abouchadi, A., Taoufik, H., Nacir, O. and Arrob, A. (2018) Pan-Facial Fractures: A Retrospective Study and Review of Literature. Open Journal of Stomatology, 8, 110-119.

https://doi.org/10.4236/ojst.2018.84010

Received: February 27, 2018

Accepted: April 15, 2018

Published: April 18, 2018

Copyright $\odot 2018$ by authors and Scientific Research Publishing Inc. This work is licensed under the Creative Commons Attribution International License (CC BY 4.0).

http://creativecommons.org/licenses/by/4.0/

(c) (i) Open Access

\begin{abstract}
Purpose: The aim of this retrospective study was to analyze the characteristics of panfacial fractures and evaluate treatment results at the Maxillofacial, Stomatology and plastic surgery department at the AVICENNE military hospital over a period of 5 years. Patients and Methods: Forty eight patients with panfacial fractures were treated in Maxillofacial, stomatology and plastic surgery department of the AVICENNE Military Teaching Hospital between 2012 and 2017. The criteria for inclusion in the study were patients who had fractures of at least three of the four axial segments of the facial skeleton: frontal, upper midface, lower midface, and mandible. Results: 48 patients with panfacial fractures had a total of 116 subtypes of facial bone fractures. A total of seventeen (14.6\%) LeFort II fractures in $16(33.4 \%)$ patients were recorded, fifteen LeFort I fractures were recorded in 3 (6.2\%) cases; seven (6\%) LeFort III fractures were recorded in $5(10.4 \%)$ cases, thirteen $(11.2 \%)$ fractures of the NOF complex were recorded in $6(12.5 \%)$ patients; sixteen $(33.4 \%)$ patients had thirty eight (32.7\%) fractures involving the mandible. Ten (8.6\%) NOM (naso-orbito-maxilla) complex fractures occurred in 9 (18.7\%) cases. $5(10.4 \%)$ patients had a total of five (4.3\%) CNEMFO (naso-ethmoido-maxillo-frontoorbital) complex fractures. Our case series included five Comminuted premaxillary fractures and six Intermaxillary disjunctions. All 48 cases had facial deformities and thirty six had malocclusions. The treatment plan to reduce and fix the facial bone fractures was sequenced "Bottom up, Outside in". Postoperative complications were reported, there were 5 cases whose malocclusions, 4 cases of zygomatic non-union or partial defects, 13 had enophthalmos and hypoglobus. Seven had scars from the trauma, 2 had lower eyelid ectropion, and 2 had temporal muscle atrophy. Conclusion: Panfacial fractures seem to be complex and difficult to treat, but with an organized and flexible approach, appropriate reduction of fractures is accomplishable, yet post-surgical complications mainly caused by soft tissue problems, including lacerations and asymmetries, can't be easily avoided.
\end{abstract}




\section{Keywords}

Panfacial, Trauma, Fracture, Treatment, Complications

\section{Introduction}

Panfacial fractures are defined as those that simultaneously involve the upper, mid and lower face [1] [2]. There is no clear definition and classification for panfacial fractures in the literature. Panfacial fractures, as defined by Follmar et al. are fracture patterns that involve at least three of the four axial segments of the facial skeleton: frontal, upper midface, lower midface, and mandible [3] (Figure 1).

Panfacial fractures are due to road traffic accidents, interpersonal violence, sports-related accidents, industrial accidents, and gunshot wounds. The mechanism of injury helps identify the energy of impact as well as the probable extent of injury [4].

Panfacial trauma is commonly associated with Multisystem injury; thus, treatment is often multidisciplinary. When the patient is stabilized, early and total restoration of facial form and function should be the goal. The management of panfacial trauma went from a conservative, delayed, multiple-staged surgery to early, aggressive, and one-stage process. High resolution computed tomography (CT), sufficient surgical exposure, proper anatomic reduction, rigid fixation, primary bone grafting, and soft tissue suspension are the basics for optimum results [4] [5].

The goal of management of panfacial fractures is the restoration of the function and aesthetic three-dimensional facial contours, the earliest possible, while minimizing the patient pain at the lowest possible cost to the victim and society. However, the ideal sequencing of a complex panfacial trauma remains the greatest challenge to every maxillo facial surgeon [6]. Fracture dislocation and the degree of comminution are decisive guidelines in the choice of the surgical procedures [7] [8]. In published literature, two classic approaches have been described for the management of panfacial trauma; namely "bottom up and inside out" or "top down and outside in". The preferred sequence starts with mandibular reconstruction, including fractures of the temporo-mandibular joints. In the next step, the fronto-facial and zygomatico-orbital compartments are reconstructed; these are key for subsequent midfacial reconstruction [8] [9].

This paper analyzes the characteristics of panfacial fractures and evaluates treatment results of a series of cases treated at AVICENNE teaching military hospital over a period of 5 years. The outcome is then discussed in the light of preexisting literature.

\section{Patients and Methods}

The study group consisted of 48 patients who had panfacial fractures and treated between January 2012 and July 2017 in the Maxillofacial Trauma Center at 


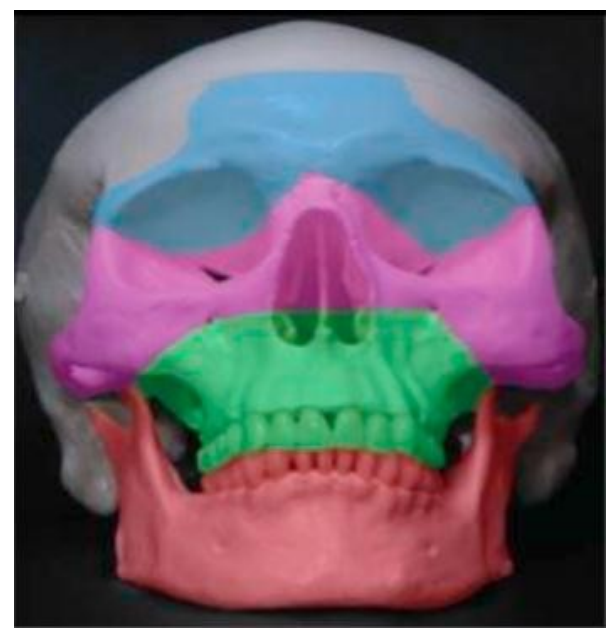

Figure 1. Four axial segments of facial skeleton [1]: Upper face: Frontal bone and superior orbital rim, Midface: Inferior orbital rim, nasal bone and zygomatic arch + Maxilla, Lower face: Mandible.

AVICENNE military hospital. The criteria for inclusion in the study were patients who had fractures of at least three of the four axial segments of the facial skeleton: frontal, upper midface, lower midface, and mandible. All of our patient had a CT (Computed Tomography) scan with 3D reconstruction for precise identification of the fractures. The 48 patients with panfacial features had a total of 116 subtypes of facial bone fractures. Road traffic accidents were the predominant etiology (71\%) (Table 1).

A retrospective chart review was carried out. Demographic information, details of treatment, and results of follow-up were tabulated for descriptive analysis. The protocol used in their management was also retrospectively analyzed. The study was approved by an ethics committee and patients' consent was taken.

\section{Results}

The 48 patients with panfacial fractures had a total of 78 subtypes of facial bone fractures. Road traffic accidents were the predominant etiology (71\%). There were 43 male and 5 female (Sex Ratio $\approx 9 / 1$ ), aged between 13 and 58 years with a main age of 34 years, 30 of them were military patients, 18 were civilians.

All patients (100\%) had midface fractures in different combinations (Table 2) (Figure 2).

A total of 17 (14.6\%) LeFort II fractures in 16 (33.4\%) patients were recorded; 10 patients had bilateral and 6 had unilateral fractures. Fifteen (13\%) LeFort I fractures were recorded in $3(6.2 \%)$ cases; 2 patients had bilateral fractures and 1 had unilateral fracture. Seven (6\%) LeFort III fractures were recorded in 5 (10.4\%) cases; 3 patients had bilateral and 2 had unilateral fractures. Thirteen 
(11.2\%) fractures of the NOF (naso-orbito-frontal) complex were recorded in 6 (12.5\%) patients. Ten (8.6\%) NOM (naso-orbito-maxilla) complex fractures occurred in $9(18.7 \%)$ cases. $5(10.4 \%)$ patients had a total of five $(4.3 \%)$ CNEMFO (naso-ethmoido-maxillo-fronto-orbital) complex fractures.

Sixteen (33.4\%) patients had thirty eight (32.7\%) fractures involving the mandible (Figure 2). 11 parasymphysis fractures were recorded in 6 patients; 4 patients had bilateral and 2 had unilateral fractures. A total of 9 condylar fractures were recorded in 4 patients; 2 patients each had unilateral and bilateral fractures. 16 angle fractures were recorded; 3 patients had bilateral fracture.

All 48 cases had facial deformities (Figure 3 ) and some had functional disorders, in our study thirty six (75\%) had malocclusions. Twenty six cases had limitation of mouth opening (54.17\%) to under $30 \mathrm{~mm}$, among which 9 had TMJ (Temporomandibular Joint) ankylosis with a range of mouth opening of less than $15 \mathrm{~mm}$. Thirteen cases (27\%) had enophthalmos or hypoglobus and three

Table 1. Circumstances of the trauma for our case series.

\begin{tabular}{llc}
\hline & \multicolumn{2}{c}{ Patients } \\
\cline { 2 - 3 } Circumstances of the trauma: & Number & Percentage (\%) \\
\hline Road traffic accidents & 34 cases & $71 \%$ \\
Falls: & & \\
- 1 accidental fall of the 5th floor & 3 cases & \\
- 1 fall of the 2nd floor in an epileptic context & & $16.25 \%$ \\
- 1syncope & 8 cases & $2 \%$ \\
Brawls or aggressions & 1 case & $4.17 \%$ \\
Ballistic trauma due to a suicide attempt (Figure 4) & 2 cases & \\
Trauma by hoofbeats & & \\
\hline
\end{tabular}

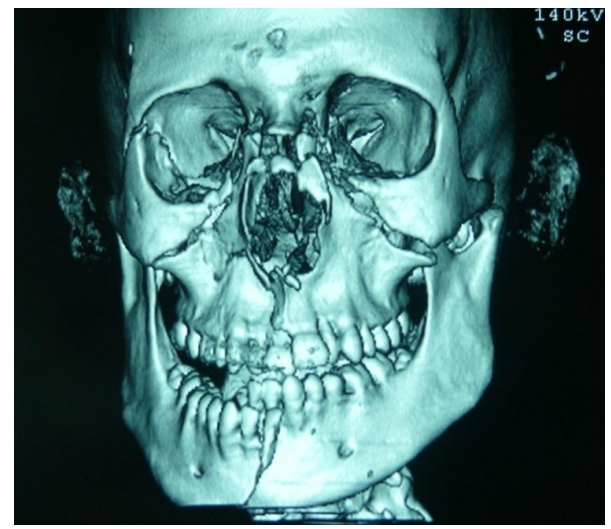

Figure 2. Panfacial fracture scannography showing mandibular symphisal fracture, bilateral zygomatic fracture with a right lateral orbital wall fracture, mediomaxillar fracture and communited nasal bone fracture. 
Table 2. Different fractures were seen in our caseseries.

\begin{tabular}{|c|c|c|c|c|c|}
\hline \multirow{2}{*}{\multicolumn{2}{|c|}{ Type of fracture }} & \multirow{2}{*}{$\frac{\text { Fractures }}{\text { Number }}$} & \multicolumn{3}{|c|}{ Patients } \\
\hline & & & Percentage (\%) & Number & Percentage (\%) \\
\hline \multicolumn{2}{|c|}{ CNEMFO } & 5 & 4.3 & 5 & 10.4 \\
\hline & Unilateral & \multirow{2}{*}{15} & \multirow{2}{*}{13} & 1 & \multirow[b]{2}{*}{6.2} \\
\hline LeFort I & Bilateral & & & 2 & \\
\hline & Unilateral & \multirow[b]{2}{*}{17} & \multirow[b]{2}{*}{14.6} & 6 & \multirow[b]{2}{*}{33.4} \\
\hline LeFort II & Bilateral & & & 10 & \\
\hline & Unilateral & \multirow[b]{2}{*}{7} & \multirow[b]{2}{*}{6} & 2 & \multirow[b]{2}{*}{10.4} \\
\hline LeFort III & Bilateral & & & 3 & \\
\hline $\mathrm{NOM}$ & $\begin{array}{c}\text { Unilateral } \\
\text { Bilateral }\end{array}$ & 10 & 8.6 & 9 & 18.7 \\
\hline \multicolumn{2}{|c|}{ NOF Complex } & 13 & 11.2 & 6 & 12.5 \\
\hline \multicolumn{2}{|c|}{ Comminuted premaxillary } & 5 & 4.3 & 2 & 4.2 \\
\hline \multirow{3}{*}{\multicolumn{2}{|c|}{ Intermaxillary disjunctions }} & 6 & 5.2 & 1 & 2 \\
\hline & & 38 & & & \\
\hline & & 2 & & & \\
\hline \multicolumn{2}{|c|}{ Mandible } & 9 & & & \\
\hline \multicolumn{2}{|c|}{ Symphysis Condyle } & 1 & & & \\
\hline \multicolumn{2}{|c|}{ Unilateral Bilateral } & 4 & & & \\
\hline \multicolumn{2}{|c|}{ Parasymphysis } & 11 & 32.7 & 16 & 33.4 \\
\hline \multicolumn{2}{|c|}{ Unilateral Bilateral } & 3 & & & \\
\hline \multicolumn{2}{|c|}{ Angle } & 4 & & & \\
\hline \multirow{3}{*}{\multicolumn{2}{|c|}{ Unilateral Bilateral }} & 16 & & & \\
\hline & & 4 & & & \\
\hline & & 6 & & & \\
\hline \multicolumn{2}{|c|}{ Total } & 116 & 100 & 48 & 100 \\
\hline
\end{tabular}

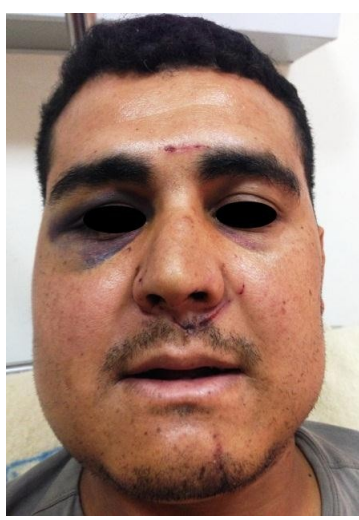

Figure 3. Panfacial fracture appearance: swelling, periorbital ecchymosis, flattening of the malar prominence, enophtalmos.

(6.25\%) had 1 globe removed before treatment of their maxillofacial injuries (Table 3).

Postoperative complications were not easily avoided; most of our patients 


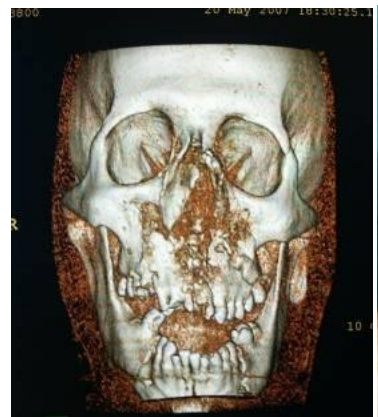

(a)

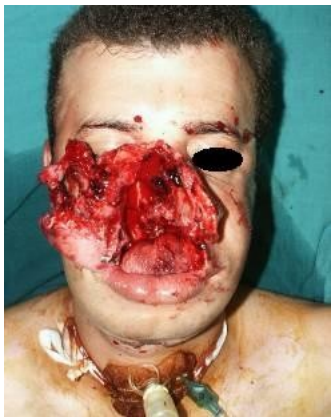

(b)

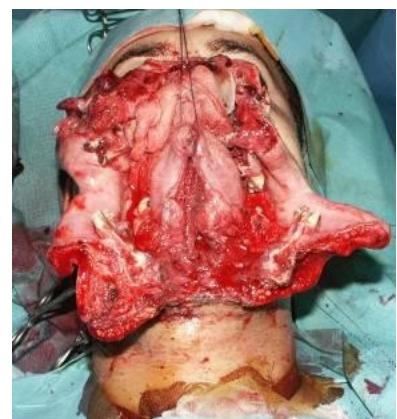

(c)

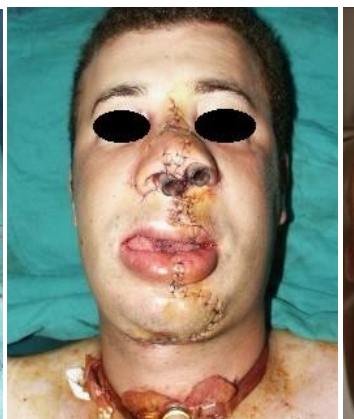

(d)

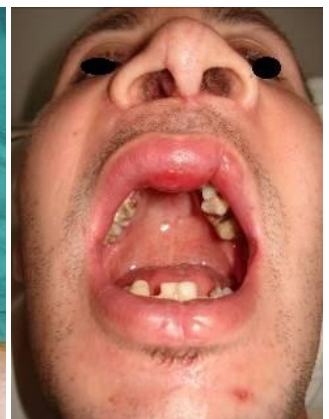

(e)

Figure 4. A 31 year old patient presenting a ballistic injury due to a suicide attempt causing an open panfacial trauma. (a) Scannographic image showing 3 staged facial fractures; (b) Pre-operative evaluation, open fractures of the nasal bone and maxilla with soft tissuesuffering; (c) Per operative procedure starting "bottom, up"; (d) Immediate post-operative result; (e) Long term post-operative evaluation: satisfying mouth opening and good occlusion.

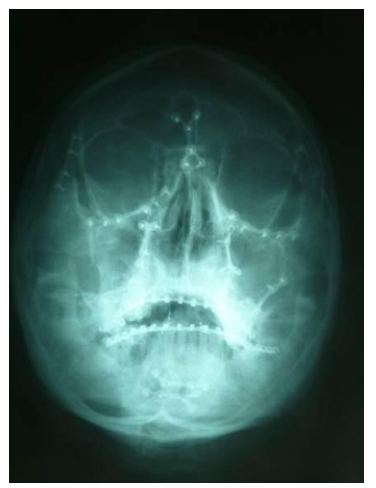

Figure 5. Post-operative osteosynthesis control on a Blondeau radiography.

suffered from esthetic and functional problems in the 3 and 6 months follow up (Table 4). Esthetic problems were at the lead of all complications: Soft tissue problems (23\%) and asymmetry (16.7\%). Patients also suffered from functional issues, 5 had malocclusion (10.4\%), 5 had mandibular hypomotility (10.4\%) and 3 had enophtalmos (6.25\%). Infections were seen in 3 of our patients $(6.25 \%)$ and were treated by antibiotics. Non-union occurred in 4 cases (8.34\%).

No intra-operative complications took place for any patient. Postoperatively, 
Table 3. Clinical findings in 48 panfacial fracture cases.

\begin{tabular}{ccc}
\hline Symptoms & Patients (n) & Percentage \% \\
\hline Facial deformity & 48 & 100 \\
Malocclusion & 36 & 75 \\
Enophthalmos/hypoglobus & 13 & 27 \\
One globe removed & 3 & 6.25 \\
Mouth opening limitation & 26 & 54.17 \\
\hline
\end{tabular}

Table 4. Postoperative complications.

\begin{tabular}{ccc}
\hline Complications & Number & $\%$ \\
\hline Malocclusion & 5 & 10.4 \\
Non-union & 4 & 8.34 \\
Infection & 3 & 6.25 \\
Enophthalmos & 3 & 6.25 \\
Asymmetry & 8 & 16.7 \\
Mandibular hypomotility & 5 & 10.4 \\
Soft tissue problems & 11 & 23 \\
\hline
\end{tabular}

control radiographs noted adequate reduction (Figure 5) and after resolution of the edema, mouth opening and mandibular movements were fairly adequate.

\section{Discussion}

There is no accepted definition of panfacial fracture in the literature. Some authors define it as fracture patterns involving both midface and mandible. Others think it must involve the upper, middle, and lower face that means the NOE complex; zygomatic complex, Le Fort midfacial area, and the mandible are all simultaneously fractured. In addition to the possible facial deformities, malocclusion and limited facial movement, panfacial trauma can impact the patient's psychological state [10].

Panfacial fractures are often associated with soft tissue injuries and loss of bone structures that can lead to severe post-traumatic deformities and disabilities. Planning the treatment of panfacial fracture is a challenging process. The timing of operative management remains controversial. Multisystem injury is commonly associated; therefore the treatment is often multidisciplinary. Fracture dislocation and the degree of comminution are decisive guidelines in the choice of the surgical procedures. Early management of fractures facilitates reduction and avoids the damage of soft tissues.

In our study, the average time of maxillofacial correction was 9 days since the majority of our patients had associated systemic or neurologic injuries; time was also needed for the edema to be resolved. All of our patients received oral corticosteroids for a quicker edema reduction; antibiotics were also given to prevent 
infection. Open fractures are considered to be an emergency, which makes the treatment planning of panfacial fracture very challenging.

A CT scanning with coronal cut was achieved from the cranium to the submental region to all of our patients for a good diagnosis. Three-dimensional facial models are also very helpful in treating delayed panfacial fractures. They give the surgeon an appreciation of the spatial relationships of the displaced skeletal components and help plan treatment.

In the OR, nasotracheal intubation using fibroscopy was used in the majority of our cases, it is a reasonable way for establishing and maintaining an airway when treating panfacial fractures with an intact nasal bone, in other cases orotracheal intubation was used in patients with teeth loss; the probe fixated in the area of the lost teeth. When IMF (Inter Maxillary Fixation) was needed, it was done post operatively after extubation. Tracheotomy was necessary in one case of ballistic injury where the nasal bone was completely destructed (Figure 4). Ramanujamet et al. believe that submental intubation is a safer, effective and time efficient method for securing the airway [11].

The treatment was based on an open reduction with titanium mini plate osteosynthesis. Multiple surgical approaches were used in accordance with the fractured bones. Most cases needed IMF, liquid alimentation of variant durations (30 to 45 days) and reeducation. A bone graft taken from the iliac crest was necessary for bone reconstruction in a number of cases where bone defect was important to stabilize the bone structure and reestablish the buttresses.

Some authors recommend that surgical correction of facial fractures be performed immediately after completion of cranial repairs. They advocate the reduction and fixation of complex injuries within $48 \mathrm{~h}$ when initial edema has resolved and a thorough clinical and radiological exam has been completed. For patients who are medically unstable because of associated neurologic or systemic injuries, facial fracture repair may have to be delayed beyond a reasonable time. A delay of 2 weeks for definitive repair increases the difficulty in obtaining adequate reduction of fracture dislocations. Carr and Mathog believe bone healing beyond 3 weeks is in a "grey stage" - the edges of the fragment begin to absorb and remodel, which makes it very difficult to obtain anatomic reduction. This can lead to bone malunion, delayed union, nonunion, and bone defect. Quick management is also critical within 10 days because soft-tissue stiffening and interfragmentary healing make delayed corrections very difficult [12].

A review of published literature on panfacial fractures revealed that two approaches of management have enjoyed universal acceptance; "top-to-bottom" and "bottom-to-top" [13] [14]. Marciani et al. [9] recommend first restoring the occlusion and alveolar ridge continuity and alignment and then repairing the mandibular body and angle fractures. Next, the vertical height of the mandibular condyles and ramus is established. They advocate exposing all mid-face and upper face fractures to allow good visualization of the fracture segments. Then, the transverse width of the face is restored by using the zygomatic arches as a guide. 
The vertical height of the face is restored by aligning and fixing all facial vertical buttresses. The continuity of the orbital and sinus floors and walls is reestablished and finally the nasoethmoidal fractures are aligned and fixed.

At our center, our approach to reduce and fix the facial bone fractures is "Bottom up, Outside in". Facial bone fractures were reduced first and then fixed in a sequential manner. The mandible was reconstructed to establish a stable base. Next, the maxilla was guided into occlusion using the intact mandible as reference and IMF was done. After simultaneously visualizing all fracture sites, the midface fractures were reduced using the "Outside in" principle. The ZMC unit was fixed first and if indicated, the zygomatic arch was lifted into position. Finally the nasal bone was reduced.

Overall, some patients had postoperative functional problems: visual and oculomotor disorders, sensory disturbances of the orbital nerve (V2) territory, morphologic deformities, limitation of oral opening, articular and dental disorders. Hence, reeducation is a weighty treatment chapter for optimal results. Moreover, aesthetic sequelae is a painful experience that every panfacial trauma victim goes through; consequently psychological support should also be provided.

On the whole, satisfaction rate of $41 / 48$ patients, most of our patients wished to be operated for removal of osteosynthesis material and/or for rhinoplasty.

\section{Conclusion}

Management of panfacial trauma allows proper restoration of facial form and function. Panfacial fractures seem to be complex and difficult to treat, but with an organized and flexible approach, appropriate reduction of fractures is accomplishable, yet post-surgical complications can't be easily avoided.

\section{References}

[1] Yang, R., Zhang, C., Liu, Y., Li, Z. and Li, Z. (2012) Why Should We Start from Mandibular Fractures in the Treatment of Panfacial Fractures? Journal of Oral and Maxillofacial Surgery, 70, 1386-1392. https://doi.org/10.1016/j.joms.2011.11.006

[2] Wenig, B.L. (1991) Management of Panfacial Fractures. Otolaryngologic Clinics of North America, 24, 93-101.

[3] Follmar, K.E., DeBruijn, M., Baccarani, A., Bruno, A.D., Mukundan, S., Erdmann, D., et al. (2007) Concomitant Injuries in Patients with Panfacial Fractures. Journal of Trauma and Acute Care Surgery, 63, 831-835. https://doi.org/10.1097/TA.0b013e3181492f41

[4] Louis, P. (2004) Management of Panfacial Fractures. In: Miloro, M., Ed., Peterson's Principles of Oral and Maxillofacial Surgery, 2nd Edition, BCDecker Inc., Hamilton.

[5] David, D.J. (1999) Maxillofacial Trauma: Principles of Management, Priorities and Basic Techniques. Trauma, 1, 215-226. https://doi.org/10.1177/146040869900100305

[6] Fritz, M.A. and Koltai, .PJ. (2002) Sequencing and Organization of the Repair of Panfacial Fractures. Operative Techniques in Otolaryngology-Head and Neck Surgery, 13, 261-264. https://doi.org/10.1016/S1043-1810(02)80056-2 
[7] He, D., Zhang, Y. and Ellis, E. (2007) Panfacial Fractures: Analysis of 33 Cases Treated Late. Journal of Oral and Maxillofacial Surgery, 65, 2459-2465. https://doi.org/10.1016/j.joms.2007.06.625

[8] Hardt, N. and Kuttenberger, J. (2010) Surgical Strategy for Complex Craniofacial Fractures. Craniofacial Trauma. Springer-Verlag, Berlin, 205-238. https://doi.org/10.1007/978-3-540-33041-7_12

[9] Marciani, R. (2009) Integrating the Care and Treatment of the Complex Facialtrauma Patient. In: Fonseca, R., Marciani, R., Eds., Oral and Maxillofacial Surgery, 2nd Edition, Saunders/Elsevier, 395-411.

[10] Tang, W., Feng, F., Long, J., Lin, Y., Wang, H., Liu, L., et al. (2009) Sequential Surgical Treatment for Panfacial Fractures and Significance of Biological Osteosynthesis. Dental Traumatology, 25, 171-175. https://doi.org/10.1111/j.1600-9657.2008.00739.x

[11] Ramanujam, L., Sehgal, S., Krishnappa, R. and Prasad, K. (2013) Panfacial Fractures-A Retrospective Analysis at MS Ramaiah Group of Hospitals, Bangalore. Journal of Oral and Maxillofacial Surgery, Medicine, and Pathology, 25, 333-340. https://doi.org/10.1016/j.ajoms.2013.02.006

[12] Carr, R.M. and Mathog, R.H. (1997) Early and Delayed Repair of Orbitozygomatic Complex Fractures. Journal of Oral and Maxillofacial Surgery, 55, 253-258. https://doi.org/10.1016/S0278-2391(97)90537-1

[13] Powers, D.B., Will, M.J., Bourgeois, S.L. and Hatt, H.D. (2005) Maxillofacial Trauma Treatment Protocol. Oral \& Maxillofacial Surgery Clinics of North America, 17, 341-355. https://doi.org/10.1016/j.coms.2005.05.003

[14] Bagheri, S.C., Khan, H.A., Jahangirnia, A., Rad, S.S. and Mortazavi, H. (2012) An Analysis of 101 Primary Cosmetic Rhinoplasties. Journal of Oral and Maxillofacial Surgery, 70, 902-909. https://doi.org/10.1016/j.joms.2011.02.075 